\title{
HESS upper limit on the very high energy $\gamma$-ray emission from the globular cluster 47 Tucanae (Research Note)
}

F. Aharonian ${ }^{1,2}$, A. G. Akhperjanian ${ }^{3}$, G. Anton ${ }^{4}$, U. Barres de Almeida ${ }^{5, \star}$, A. R. Bazer-Bachi ${ }^{6}$, Y. Becherini ${ }^{7}$, B. Behera ${ }^{8}$, K. Bernlöhr 1,9 , C. Boisson ${ }^{10}$, A. Bochow ${ }^{1}$, V. Borrel ${ }^{6}$, I. Braun ${ }^{1}$, E. Brion ${ }^{11}$, J. Brucker ${ }^{4}$, P. Brun ${ }^{11}$, R. Bühler ${ }^{1}$, T. Bulik ${ }^{12}$, I. Büsching ${ }^{13}$, T. Boutelier ${ }^{14}$, P. M. Chadwick ${ }^{5}$, A. Charbonnier ${ }^{15}$, R. C. G. Chaves ${ }^{1}$, A. Cheesebrough ${ }^{5}$, L.-M. Chounet ${ }^{16}$, A. C. Clapson $^{1}$, G. Coignet $^{17}$, M. Dalton ${ }^{9}$, M. K. Daniel ${ }^{5}$, I. D. Davids ${ }^{18,13}$, B. Degrange ${ }^{16}$, C. Deil ${ }^{1}$, H. J. Dickinson ${ }^{5}$, A. Djannati-Atai ${ }^{7}$, W. Domainko ${ }^{1}$, L. O'C. Drury ${ }^{2}$, F. Dubois ${ }^{17}$, G. Dubus ${ }^{14}$, J. Dyks ${ }^{12}$, M. Dyrda ${ }^{19}$, K. Egberts ${ }^{1}$, D. Emmanoulopoulos ${ }^{8}$, P. Espigat ${ }^{7}$, C. Farnier $^{20}$, F. Feinstein ${ }^{20}$, A. Fiasson ${ }^{20}$, A. Förster ${ }^{1}$, G. Fontaine ${ }^{16}$, M. Füßling 9 , S. Gabici ${ }^{2}$, Y. A. Gallant ${ }^{20}$, L. Gérard ${ }^{7}$, B. Giebels ${ }^{16}$, J. F. Glicenstein ${ }^{11}$, B. Glück ${ }^{4}$, P. Goret ${ }^{11}$, D. Hauser ${ }^{8}$, M. Hauser ${ }^{8}$, S. Heinz ${ }^{4}$, G. Heinzelmann ${ }^{21}$, G. Henri ${ }^{14}$, G. Hermann ${ }^{1}$, J. A. Hinton ${ }^{22}$, A. Hoffmann ${ }^{23}$, W. Hofmann ${ }^{1}$, M. Holleran ${ }^{13}$, S. Hoppe ${ }^{1}$, D. Horns ${ }^{21}$, A. Jacholkowska ${ }^{15}$, O. C. de Jager ${ }^{13}$, I. Jung ${ }^{4}$,

K. Katarzyńskii ${ }^{24}$, U. Katz ${ }^{4}$, S. Kaufmann ${ }^{8}$, E. Kendziorra ${ }^{23}$, M. Kerschhaggl ${ }^{9}$, D. Khangulyan ${ }^{1}$, B. Khélifi ${ }^{16}$, D.

$\mathrm{Keogh}^{5}$, Nu. Komin ${ }^{11}$, K. Kosack ${ }^{1}$, G. Lamanna ${ }^{17}$, J.-P. Lenain ${ }^{10}$, T. Lohse ${ }^{9}$, V. Marandon ${ }^{7}$, J. M. Martin ${ }^{10}$, O. Martineau-Huynh ${ }^{15}$, A. Marcowith ${ }^{20}$, D. Maurin ${ }^{15}$, T. J. L. McComb ${ }^{5}$, M. C. Medina ${ }^{10}$, R. Moderski ${ }^{12}$, E. Moulin ${ }^{11}$, M. Naumann-Godo ${ }^{16}$, M. de Naurois ${ }^{15}$, D. Nedbal ${ }^{25}$, D. Nekrassov ${ }^{1}$, J. Niemiec ${ }^{19}$, S. J. Nolan ${ }^{5}$, S. Ohm ${ }^{1}$, J.-F. Olive ${ }^{6}$, E. de Oña Wilhelmi ${ }^{7,26}$, K. J. Orford ${ }^{5}$, M. Ostrowski ${ }^{27}$, M. Panter ${ }^{1}$, M. Paz Arribas ${ }^{9}$, G. Pedaletti ${ }^{8}$, G. Pelletier ${ }^{14}$, P.-O. Petrucci' ${ }^{14}$, S. Pita ${ }^{7}$, G. Pühlhofer ${ }^{8}$, M. Punch ${ }^{7}$, A. Quirrenbach ${ }^{8}$, B. C. Raubenheimer ${ }^{13}$, M. Raue ${ }^{1,26}$,

S. M. Rayner ${ }^{5}$, O. Reimer ${ }^{28}$, M. Renaud ${ }^{7,1}$, F. Rieger ${ }^{1,26}$, J. Ripken ${ }^{21}$, L. Rob ${ }^{25}$, S. Rosier-Lees ${ }^{17}$, G. Rowell ${ }^{29}$, B. Rudak ${ }^{12}$, C. B. Rulten ${ }^{5}$, J. Ruppel ${ }^{30}$, V. Sahakian ${ }^{3}$, A. Santangelo ${ }^{23}$, R. Schlickeiser ${ }^{30}$, F. M. Schöck ${ }^{4}$, R. Schröder ${ }^{30}$, U. Schwanke ${ }^{9}$, S. Schwarzburg ${ }^{23}$, S. Schwemmer ${ }^{8}$, A. Shalchi ${ }^{30}$, J. L. Skilton ${ }^{22}$, H. Sol ${ }^{10}$, D. Spangler ${ }^{5}$, Ł. Stawarz ${ }^{27}$, R. Steenkamp ${ }^{18}$, C. Stegmann ${ }^{4}$, G. Superina ${ }^{16}$, A. Szostek ${ }^{1}$, P. H. Tam ${ }^{8}$, J.-P. Tavernet ${ }^{15}$, R. Terrier ${ }^{7}$, O. Tibolla ${ }^{1,8}$, C. van Eldik ${ }^{1}$, G. Vasileiadis ${ }^{20}$, C. Venter ${ }^{13}$, L. Venter ${ }^{10}$, J. P. Vialle ${ }^{17}$, P. Vincent ${ }^{15}$, M. Vivier ${ }^{11}$, H. J. Völk ${ }^{1}$, F. Volpe ${ }^{1,16,26}$, S. J. Wagner ${ }^{8}$, M. Ward ${ }^{5}$, A. A. Zdziarski ${ }^{12}$, and A. Zech ${ }^{10}$

(Affiliations can be found after the references)

Received 22 December 2008 / Accepted 15 March 2009

\section{ABSTRACT}

Observations of the globular cluster 47 Tucanae (NGC 104), which contains at least $23 \mathrm{~ms}$ pulsars, were performed with the HESS telescope system. The observations lead to an upper limit of $F(E>800 \mathrm{GeV})<6.7 \times 10^{-13} \mathrm{~cm}^{-2} \mathrm{~s}^{-1}$ on the integral $\gamma$-ray photon flux from 47 Tucanae. Considering millisecond pulsars as the unique potential source of $\gamma$-rays in the globular cluster, constraints based on emission models are derived: on the magnetic field in the average pulsar nebula and on the conversion efficiency of spin-down power to $\gamma$-ray photons or to relativistic leptons.

Key words. Galaxy: globular clusters: individual: 47 Tucanae - stars: pulsars: general - gamma rays: observations

\section{Introduction}

Millisecond pulsars (msPSRs) are usually categorized among the radio pulsar population by limits on their spin period $(P \leq$ $50 \mathrm{~ms})$ and, when available, intrinsic spin-down rate $\left(\dot{P}_{\text {int }} \leq\right.$ $\left.10^{-18} \mathrm{~s} \mathrm{~s}^{-1}\right)$. They are old neutron stars, possibly re-accelerated by interactions with a companion, as first proposed in Alpar et al. (1982). Very high energy (VHE) emission from this type of object has been predicted via various radiation mechanisms. For individual objects, Inverse Compton (IC) or Curvature Radiation (CR) emission due to the acceleration of leptons above the polar cap (Harding et al. 2005; Bulik et al. 2000) have been proposed. For binary systems, an additional possibility would be the interaction between pulsar wind driven outflows and the stellar wind

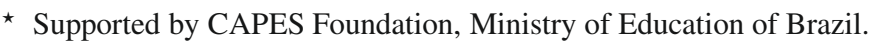

of the companion (see for instance Dubus 2006). The spin-down power, typically lower than $10^{35} \mathrm{erg} \mathrm{s}^{-1}$, entails expected individual $\gamma$-ray fluxes well below the detection threshold of current instruments.

However, groups of msPSRs have been identified in Galactic globular clusters (see e.g. Manchester et al. 1991), allowing for larger fluxes from an ensemble of unresolved sources. Out of more than 185 pulsars with $P \leq 50 \mathrm{~ms}$ known in the year $2008^{1}$ (Manchester et al. 2005), 131 belong to globular clusters ${ }^{2}$. Globular clusters (GCs) are old high-density galactic structures, with ages close to the age of the Galaxy itself (see for instance

\footnotetext{
1 http://www.atnf.csiro.au/research/pulsar/psrcat/ [v1.34].

2 http://www2 naic. edu/ pfreire/GCpsr.html [on 2008 August 7].
} 
Gratton et al. 2003). Their age indeed suggests evolved embedded stellar populations including compact (binary) objects, which are considered potential progenitors to msPSRs, as discussed e.g. in Benaquista (2006). The GCs Terzan 5, 47 Tucanae and M 28, in this order, host the largest identified msPSR populations (Ransom 2008).

47 Tucanae (NGC 104) is one of the largest Galactic GCs known to date, with an estimated mass of $10^{6} M_{\odot}$ and an age of $11.2 \pm 1.1$ Gyr (Gratton et al. 2003). Optical observations by the Hubble Space Telescope, described in McLaughlin et al. (2006), allowed precise measurements of its location, centered at $\alpha_{2000}=0^{\mathrm{h}} 24^{\mathrm{m}} 05^{\mathrm{s}} .67$ and $\delta_{2000}=-72^{\circ} 04^{\prime} 52^{\prime \prime} .62$ and placed it at a distance of $4.0 \pm 0.35 \mathrm{kpc}$. The surface brightness distribution allows the estimation of a core radius of $r_{0}=20^{\prime} .84 \pm 5^{\prime \prime}$. 05 , a half-mass radius $r_{\mathrm{h}} \approx 2.6^{\prime}$ and a tidal radius $r_{\mathrm{t}} \approx 0.6^{\circ}$, using the model of King (1966). In 47 Tucanae, 23 pulsars so far were revealed, with radio observations predominantly using the Parkes telescope (Freire et al. 2003), with periods in the range 2-8 ms, averaging at $4 \mathrm{~ms}$, all located within $1.2^{\prime}$ of the centre of the GC. Based on the unresolved $20 \mathrm{~cm}$ radio flux from the core of 47 Tucanae, McConnell et al. (2004) estimated that up to 30 pulsars could be radio-detected. A study of the dispersion measure of the observed pulsar period derivatives (Freire et al. 2001) provided an estimation of the average msPSR intrinsic period derivative with $\langle\dot{P} / P\rangle_{\text {int }} \approx 10^{-18} \mathrm{~s}^{-1}$ and hence a surface dipole magnetic field $B_{\mathrm{s}} \approx 2.6 \times 10^{8} \mathrm{G}$ and a spin-down power of $L_{\mathrm{sd}} \approx 10^{34} \mathrm{erg} \mathrm{s}^{-1}$.

At higher energies, Heinke et al. (2005) reported, from Chandra X-ray observatory data on 47 Tucanae, some 200 X-ray point sources, which belong to several object classes including cataclysmic variables, low-mass X-ray binaries (XRB), and the radio-detected msPSRs. They derive, from a tentative identification of the unknown sources they detected, an upper limit on the number of pulsars in the core of 47 Tucanae of about 60, assuming individual fluxes similar to the X-ray detected ones. Roughly two thirds of these msPSRs have a stellar companion $\left(M \leq 0.2 M_{\odot}\right)$. The X-ray spectrum of a msPSR in a GC can be described by a thermal component plus single power law, with typical X-ray (0.5-6 keV) fluxes around $10^{31} \mathrm{erg} \mathrm{s}^{-1}$ (Bogdanov et al. 2006). A few msPSRs exhibit X-ray pulsations, although with pulsed fractions below $50 \%$ for most of them (Cameron et al. 2007). The presence in 47 Tucanae of "hidden" msPSRs, detectable in hard X-rays but not in radio, has been excluded, within the uncertainty of the model by Tavani (1991), by the high-energy X-ray ( 0.75 to $30 \mathrm{MeV})$ upper limits reported by COMPTEL (O'Flaherty et al. 1995). From EGRET observations, Michelson et al. (1994) produced a photon flux upper limit of $5 \times 10^{-8} \mathrm{~cm}^{-2} \mathrm{~s}^{-1}$ above $100 \mathrm{MeV}$ at $95 \%$ confidence level. In the same energy band, a detection of 47 Tucanae was just announced with the release of the FGST bright source list ${ }^{3}$, see Abdo et al. (2009), slightly below the EGRET upper limit by Michelson et al. (1994). These are discussed in Sect. 3.

In the $\mathrm{TeV}$ range, previous observations of globular clusters resulted in upper limits. A limit on the steady photon flux from M 13 (5 msPSRs, $7 \mathrm{kpc}$ ) was established by the Whipple Telescope (Hall et al. 2003) at $1.08 \times 10^{-11} \mathrm{~cm}^{-2} \mathrm{~s}^{-1}$ above $500 \mathrm{GeV}$. 47 Tucanae was observed by the Durham Mark III telescopes, with a resulting upper limit on the photon flux in pulsed emission from selected pulsars of $4.4 \times 10^{-11} \mathrm{~cm}^{-2} \mathrm{~s}^{-1}$, above a threshold of $450 \mathrm{GeV}$ (Bowden et al. 1991). Periodic VHE emission from an XRB in 47 Tucanae above $5 \mathrm{TeV}$ was

\footnotetext{
http://fermi.gsfc.nasa.gov/ssc/data/access/lat/ bright_src_list/
}

reported once, by de Jager et al. (1989) during a remarkably high X-ray flux episode (Auriere et al. 1989). Such event has not been reported since then in 47 Tucanae.

The large number of identified msPSRs in 47 Tucanae and the compactness of the msPSR population at relatively close distance motivated HESS observations of this GC, to investigate the predicted VHE emission from this class of objects. The results of these observations are presented in Sect. 2. Given the unknowns regarding VHE-emitting XRB, the interpretation given in Sect. 3 centers on a collective signature from the msPSR population at $\mathrm{TeV}$ energies.

\section{Observations and analysis}

HESS is an array of four Imaging Atmospheric Cherenkov Telescopes, located in the Khomas Highland of Namibia. Stereoscopic analysis methods allow efficient background (cosmic ray) rejection and accurate energy and arrival direction reconstruction for $\gamma$-rays in the range $100 \mathrm{GeV}-100 \mathrm{TeV}$. For point-like sources, the system has a detection sensitivity of $1 \%$ of the flux level of the Crab Nebula above $1 \mathrm{TeV}$ with a significance of $5 \sigma$ in $25 \mathrm{~h}$ of observation. A thorough discussion of the HESS standard analysis and performance of the instrument can be found in Aharonian et al. (2006a).

A total of $13 \mathrm{~h}$ of 4-telescope data have been taken by HESS between October and November 2005 on 47 Tucanae (excluding data taken during bad weather or affected by hardware irregularities). The target was observed with an average zenith angle of $50^{\circ}$ and mean target offset of $1^{\circ}$ from the centre of the field of view. Applying the HESS analysis "standard" cuts for point-like sources (see Aharonian et al. 2006a), the energy threshold is about $800 \mathrm{GeV}$ and the point-spread function above $1 \mathrm{TeV}$ is $0.11^{\circ}$, too large to resolve the core of 47 Tucanae. Tighter cuts would slightly improve the sensitivity and angular resolution but also increase the energy threshold, further reducing the chances of a detection according to the models (see Sect. 3). Several methods for $\gamma$-ray reconstruction (the Hillas parameters method and a semi-analytical approach described in Rolland et al. 2004) and background estimation (the "ring" and "reflected" algorithms discussed in Aharonian et al. 2006a) were used, with consistent results.

We find no significant $\gamma$-ray event excess over the estimated background from the direction of 47 Tucanae. With standard cuts and using the "reflected" background estimation method, the significance of the excess in the $0.11^{\circ}$ radius integration area is $0.7 \sigma$. This allows us to set an upper limit on the flux from the target region. We determined upper limits according to Feldman \& Cousins (1998) with a 99\% confidence level, assuming a point-like source and a power law photon flux energy spectrum of index $\alpha=2$. The integral flux upper limit discussed here and shown in Fig. 1 was derived using the standard Hillas analysis, consistent within $20 \%$ with cross-check analyses. Increasing the photon index to $\alpha=3$ does not modify the result by more than $20 \%$. The upper limit on the integral photon flux in the HESS energy range for this data set $(800 \mathrm{GeV}-48.6 \mathrm{TeV}$, from the energy range of the collected events) is $6.7 \times 10^{-13} \mathrm{~cm}^{-2} \mathrm{~s}^{-1}$ or $\sim 2 \%$ of the Crab flux. This translates into a limit on the energy flux in the same energy range of $6.8 \times 10^{33} \mathrm{erg} \mathrm{s}^{-1}$ when placing 47 Tucanae at $4 \mathrm{kpc}$ distance. We also investigated an extended region $\left(0.2^{\circ}\right.$ radius $)$, without finding a significant excess. We do not discuss the extended case further due to the compact distribution of the msPSRs in 47 Tucanae and the generally weaker limits derived for extended regions. 


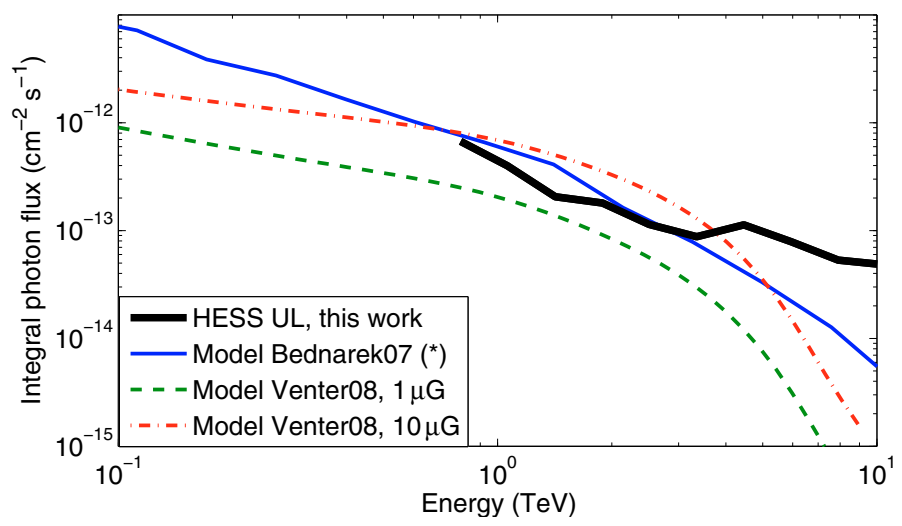

Fig. 1. Upper limit integral flux curve derived from the HESS observations of 47 Tucanae (assuming a photon index of $\alpha=2$ ), for "standard" cuts, at the 99\% confidence level. Predicted fluxes for $100 \mathrm{msPSRs}$ were added for comparison, rescaled for a distance of $\left.4 \mathrm{kpc} .{ }^{*}\right)$ Curve adapted from Bednarek \& Sitarek (2007), for $\epsilon_{\mathrm{e}}=0.01, E_{\min }=$ $100 \mathrm{GeV}$ and $\alpha=2$, rescaled to $L_{\mathrm{sd}}=10^{34} \mathrm{erg} \mathrm{s}^{-1}$ (see Sect. 3 for details).

\section{Discussion}

The HESS upper limit on the $\gamma$-ray flux emitted by 47 Tucanae can be confronted with scenarios of VHE $\gamma$-ray emission by msPSRs involving accelerated leptons in progressively larger regions: close to the pulsar, inside the pulsar wind nebula (PWN), at the boundary of the eventual PWN, or further away in the GC where pulsar winds may interact. The comparison to PWNs detected in the VHE range is also discussed. We only consider here average properties of the msPSRs in 47 Tucanae, as summarized in Sect. 1, for populations of 23 (detected) or 100 sources. While observational results favor smaller numbers, results from dynamical models of GCs, e.g. from Ivanova et al. (2008), suggest possibly larger populations. Unless stated otherwise, the following constraints scale linearly with the number of pulsars.

The production of $\gamma$-rays in the pulsar magnetosphere has been proposed in (at least) two different general scenarios, which consider different production sites: the "outer gap" or the "polar cap". In the "outer gap" model (see e.g. Chen \& Ruderman 1993), low values of the surface magnetic field (estimated from the spin-down rate) and pulsar period, which define the conditions near the light cylinder, are believed to generally prevent VHE emission from msPSRs. Although the "polar cap" model (discussed for instance in Harding et al. 2005) does not have such restriction on the conditions for VHE emission, both classes of model predict the flux to drop off sharply between 1 and $100 \mathrm{GeV}$, as discussed for a single msPSR in Chiang \& Romani (1992) and in Wang et al. (2005) for a large population. The upper limit by EGRET (Michelson et al. 1994) does constrain some of these models. Pulsed emission is also predicted, e.g. in Venter \& de Jager (2008) for 47 Tucanae, to drop before $100 \mathrm{GeV}$, below the limit by Bowden et al. (1991). The Fermi detection will undoubtedly renew the discussion on these processes, but interpretation in the $20 \mathrm{MeV}-300 \mathrm{GeV}$ band will be challenging, between potentially pulsed emission from one or more msPSR, confused or unresolved sources, and the overall steady emission component, which could be tied to scenarios also valid at energies above our quoted threshold.

The IC component, produced either in the magnetosphere or further away from the compact object, does extend to the energies considered here, but in most cases with only very low fluxes (Bulik et al. 2000). Still, when considering populations

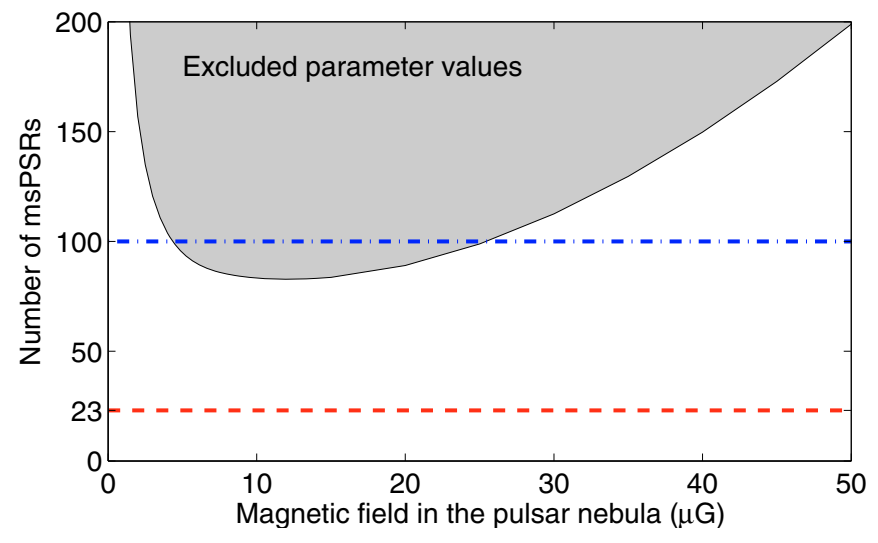

Fig. 2. Upper limit on the number of msPSRs in 47 Tucanae for a given average magnetic field in the pulsar nebula, using the model by Venter et al. (2009) and the HESS flux upper limit. The dashed lines indicate the number of observed msPSRs (23) and the 100 msPSRs hypothesis discussed here.

of sources, as done in Venter et al. (2009), IC emission from the pulsar nebulae might reach observable levels. Their Monte Carlo simulations of msPSR populations, accounting for the observed range of parameters ( $P, \dot{P}_{\text {int }}$, viewing geometry), predict the cumulative flux from 100 msPSRs, as illustrated in Fig. 1. The efficiency of the IC emission in the pulsar nebula increases with the strength of the nebular magnetic field $B$ (in relation with the increased confinement time) until losses by synchrotron radiation become dominant. For a given value of $B$, the HESS upper limit can be normalized by the predicted flux per pulsar to obtain the maximum allowed number of msPSRs, as done in Fig. 2. Large msPSR populations are thus excluded, down to 80 objects for $B=12 \mu \mathrm{G}$. In this model, the prediction falls short of providing constraints for only 23 msPSRs. Using the limit on the magnetic field strength in the nebula - post-shock - of the millisecond PSR J0437-4715 by Zavlin et al. (2002) of $B<18 \mu \mathrm{G}$ and possibly lower (see the discussion in that reference), and assuming similar properties for 100 msPSRs, this would suggest $B \leq 5 \mu \mathrm{G}$ in the average pulsar nebula.

Another scenario for producing VHE $\gamma$-ray emission relies on particle acceleration at the shock discontinuity of a PWN. Thorough discussions on pulsar winds can be found in Kaspi et al. (2006). In X-rays, pulsar wind emission from msPSRs has been observed, in the so-called "black widow" discussed in Stappers et al. (2003), with luminosities similar to those of canonical pulsars, but not in a GC. In this object, as well as for the "Mouse" pulsar (Gaensler et al. 2004), there are indications of interaction with the interstellar medium, suggesting a bow shock geometry primarily driven by the proper motion of the pulsar rather than by its accelerated particles. However, Cheng et al. (2006) established that in a GC such bow shock emission would be hampered by the geometry and stellar density. VHE $\gamma$-ray emission from several PWNs has already been detected and Cheng et al. (1986) suggested that msPSRs host the same leptonic emission processes as young pulsars like Vela $\mathrm{X}$ $\left(290 \mathrm{pc}, L_{\mathrm{sd}} \approx 10^{36} \mathrm{erg} \mathrm{s}^{-1}\right)$. Without assuming a particular emission process (see Horns et al. 2006, for a hadronic VHE emission model for the Vela X PWN), we derive the flux expected if similar objects were located in 47 Tucanae. The VHE detection of the Vela X PWN (Aharonian et al. 2006b) gives an integral photon flux $F(E>800 \mathrm{GeV}) \approx 1.5 \times 10^{-11} \mathrm{~cm}^{-2} \mathrm{~s}^{-1}$. Scaling for the distance and spin-down power of the pulsar associated with the Vela X nebula to the pulsars in 47 Tucanae amounts to a 
Table 1. Upper limits on conversion efficiencies from spin-down power.

\begin{tabular}{cccc|c}
\hline \hline \multicolumn{3}{c|}{ Model (†) $\left[E_{\min }(\mathrm{GeV}), \alpha\right]$} & Measured $\left\langle L_{\mathrm{sd}}\right\rangle$ \\
$100,2.1$ & $100,3.0$ & $1,2.1$ & $1,3.0$ & \\
\hline \multicolumn{5}{c}{$\epsilon_{\mathrm{e}}$} \\
\hline 0.003 & 0.01 & 0.01 & 0.6 & $\epsilon_{\mathrm{sd}}^{1-10}$ \\
\hline
\end{tabular}

factor $5.3 \times 10^{-5}$. We cannot constrain this model, as 840 "Velalike" msPSRs would be required to reach our flux upper limit. From pulsar properties and measured fluxes, it is usual to estimate the fraction of the spin-down power converted to $\gamma$-rays, $\epsilon_{\mathrm{sd}}$, as compiled recently in Hessels et al. (2008) for the VHEdetected PWNs in the 1-10 TeV energy band. For 47 Tucanae, this fraction is limited by $N_{\mathrm{p}} \times \epsilon_{\mathrm{sd}}^{1-10} \leq 0.7$. Any msPSR population with $N_{\mathrm{p}} \geq 23$ gives $\epsilon_{\mathrm{sd}}^{1-10}$ in the broad range of detected PWNs $\left(8 \times 10^{-5}\right.$ to 0.05$)$. From this point of view, the msPSRs in 47 Tucanae cannot be distinguished from the much younger and more energetic pulsars detected through the VHE emission of their PWN. Detailed studies of the specificities of each PWN might clarify the picture.

Nonetheless, a scenario by Bednarek \& Sitarek (2007) proposes that the energy of primary particles for the IC process increases through the interaction of the leptonic pulsar winds inside the GC. No observational evidence for such wind-wind interaction has yet been found. They predict appreciable VHE $\gamma$-ray fluxes for a population of 100 msPSRs when the power emitted by each pulsar is fixed at $1.2 \times 10^{35} \mathrm{erg} \mathrm{s}^{-1}$. The distribution in energy of the leptons produced by a pulsar is assumed to follow a power law of index $\alpha$, above a minimum energy $E_{\min }$. In most cases, the predicted flux in the HESS energy range for 47 Tucanae should be above the detection threshold of the instrument. According to this model, a non-detection translates in a limit on $N_{\mathrm{P}} \times \epsilon_{\mathrm{e}}$, the number of pulsars times the conversion efficiency from the pulsar spin-down power into relativistic electron-positron pairs (and not $\epsilon_{\mathrm{sd}}$, from spin-down to photons). The available HESS data on 47 Tucanae do not allow to reach the reference sensitivity used by Bednarek \& Sitarek (2007), estimated (for $50 \mathrm{~h}$ of observation at $20^{\circ}$ zenith angle and $0.5^{\circ}$ offset) as a photon flux of about $2.0 \times 10^{-13} \mathrm{~cm}^{-2} \mathrm{~s}^{-1}$ above $800 \mathrm{GeV}$, a factor $f_{\text {sens }} \approx 3.35$ lower than the result presented here. Besides, the values assumed in Bednarek \& Sitarek (2007) for the distance to 47 Tucanae $(4.5 \mathrm{kpc})$ and the individual spindown power $\left(1.2 \times 10^{35} \mathrm{erg} \mathrm{s}^{-1}\right)$ may be too large. Overall, a factor $f_{\text {sens }} \times\left(L_{\text {sd }}^{\text {Bednarek07 }} / L_{\text {sd }}^{\text {data }}\right) \times\left(d^{\text {data }} / d^{\text {Bednarek07 }}\right)^{2} \approx 31.8$ must be applied when comparing their model predictions to the presented HESS upper limit. Since their original limit is on $N_{\mathrm{P}} \times \epsilon_{\mathrm{e}}$, a linear rescaling can be applied when changing the number of pulsars. Rescaled conversion efficiencies, derived from the HESS upper limit above $800 \mathrm{GeV}$ assuming $100 \mathrm{msPSRs}$, are given in Table 1 for their model (noted $\ddagger$ ) and for the conversion from spin-down power to VHE emission $\left(\epsilon_{\mathrm{sd}}^{1-10}\right)$ discussed above. The comparison depends on the injection spectrum of the leptons produced by the pulsars. All the proposed scenarios are constrained $\left(\epsilon_{\mathrm{e}}<1\right)$ in the 100 msPSRs case, with most limits on the efficiency clearly below the estimated $\epsilon_{\mathrm{e}} \approx 0.1$ for the Crab nebula (Bednarek \& Sitarek 2007), even when assuming only 23 msPSRs. The exception is the scenario where most of the leptons are produced with low energy $\left(E_{\min }=1 \mathrm{GeV}\right.$ and $\alpha=3$ ): the constraints weaken to $\epsilon_{\mathrm{e}} \leq 0.6$ for $100 \mathrm{msPSR}$ and $\epsilon_{\mathrm{e}} \geq 1$ (no constraint at all) for 23 msPRSs.

\section{Conclusions}

The upper limit of the VHE $\gamma$-ray photon flux obtained from HESS observations of 47 Tucanae, $F(E>800 \mathrm{GeV})<6.7 \times$ $10^{-13} \mathrm{~cm}^{-2} \mathrm{~s}^{-1}$, is at present the second limit for a GC with a sizable population of msPSRs. Given the size of this population, it is the most constraining upper limit on the flux from an ensemble of msPSRs so far derived.

Comparing this result to emission models, we considered msPSRs as the only potential $\gamma$-ray sources in the GC. Owing to the high energy threshold of these observations, emission models for the pulsar polar region, generally predicting low fluxes at these energies, cannot be constrained, except when assuming msPSR populations much larger than considered here (23-100). These numbers, according to Venter et al. (2009), may however be sufficient for the total IC emission to reach flux levels where the number of pulsars can be limited, depending on the strength of the magnetic field in the pulsar nebula, down to $B \leq 5 \mu \mathrm{G}$ in the average pulsar nebula for 100 msPSRs. The limit on the conversion efficiency from spin-down power to VHE flux (see Table 1) is compatible with the results available for VHE-detected PWNs. Collective IC emission as proposed by Bednarek \& Sitarek (2007) cannot be more efficient than in the Crab nebula for most of their sets of parameters. Complementary constraints at lower energy should follow the detection of 47 Tucanae by the Fermi Large Area Telescope, but given the possible complexity of the emission in the $\mathrm{GeV}$ range, the connection to the VHE band cannot be assessed here.

Acknowledgements. The support of the Namibian authorities and of the University of Namibia in facilitating the construction and operation of HESS is gratefully acknowledged, as is the support by the German Ministry for Education and Research (BMBF), the Max Planck Society, the French Ministry for Research, the CNRS-IN2P3 and the Astroparticle Interdisciplinary Programme of the CNRS, the UK Science and Technology Facilities Council (STFC), the IPNP of the Charles University, the Polish Ministry of Science and Higher Education, the South African Department of Science and Technology and National Research Foundation, and by the University of Namibia. We appreciate the excellent work of the technical support staff in Berlin, Durham, Hamburg, Heidelberg, Palaiseau, Paris, Saclay, and in Namibia in the construction and operation of the equipment.

\section{References}

Abdo, A. A., et al. (Fermi LAT collaboration) [arXiv: 0902.1340v1]

Aharonian, F., Akhperjanian, A. G., Bazer-Bachi, A. R., et al. (HESS collaboration) 2006a, A\&A, 457, 899A

Aharonian, F., Akhperjanian, A. G., Bazer-Bachi, A. R., et al. (HESS collaboration) 2006b, A\&A, 448, L43

Alpar, M. A., Cheng, A. F., Ruderman, M. A., \& Shaham, J. 1982, Nature, 300, 728

Aurière, M., Koch-Miramond, L., \& Ortoloani, S. 1989, A\&A, 214, 113

Bednarek, W., \& Sitarek, J. 2007, MNRAS, 377, 920

Benaquista, M. J. 2006, Living Rev. Relativity, 9, URL (cited on May 14, 2009), http: //www . livingreviews . org/lrr-2006-2

Bogdanov, S., Grindlay, J. E., Heinke, C. O., et al. 2006, ApJ, 646, 1104

Bowden, C. C. G., et al. 1991, Proc. 22d Int. Cosmic-Ray Conf., Dublin, 1, 424 Bulik, T., Rudak, B., \& Dyks, B. 2000, MNRAS, 317, 97

Cameron, P. B., Rutledge, R. E., Camilo, F., et al. 2007, ApJ, 660, 587

Chen, K., \& Ruderman, M. 1993, ApJ, 402, 264

Cheng, K. S., Ho, C., \& Ruderman, M. 1986, ApJ, 300, 522

Cheng, K. S., Taam, R. E., \& Wang, W. 2006, ApJ, 641, 427

Chiang, J., \& Romani, R. W. 1992, ApJ, 400, 629

Dubus, G. 2006, A\&A, 456, 801D

Feldman, G. J., \& Cousins, R. D. 1998, Phys. Rev. D, 57, 3873

Freire, P. C., Kramer, M., Lyne, A. G., et al. 2001, ApJ, 557, L105

Freire, P. C., Camilo, F., Kramer, M., et al. 2003, MNRAS, 340, 1359

Gaensler, B. M., van der Swaluw, E., Camilo, F., et al. 2004, ApJ, 616, 383 
Gratton, R. G., Bragaglia, A., Carretta, E., et al. 2003, A\&A, 408, 529 Hall, T. A., Bond, I. H., Bradbury, S. M., et al. 2003, ApJ, 583, 853

Harding, A. K., Usov, V. V., \& Muslimov, A. G. 2005, ApJ, 622, 531

Heinke, C. O., Grindlay, J. E., Edmonds, P. D., et al. 2005, ApJ, 625, 796

Hessels, J. W. T., Nice, D. J., Gaensler, B. M., et al. 2008, ApJ, 682, L41

Horns, D., Aharonian, F., Santangelo, A., Hoffmann, A. I. D., \& Masterson, C. 2006, A\&A, 451, L51

Ivanova, N., Heinke, C. O., Rasio, F. A., Belczynski, K., \& Fregeau, J. M. 2008, MNRAS, 386, 553

de Jager, O. C., et al. 1989, IAU Rep., 4858

Kaspi, V. M., Roberts, M. S. E., \& Harding, A. K. 2006, Compact Stellar X-ray Sources (Cambridge University Press), 279

King, I. R. 1966, ApJ, 71, 64

Manchester, R. N., Lyne, A. G., Robinson, C., Bailes, M., \& D’Amico, N. 1991, Nature, 352, 219M

Manchester, R. N., Hobbs, G. B., Teoh, A., \& Hobbs, M. 2005, AJ, 129, 1993

McConnell, D., Deshpande, A. A., Connors, T., \& Ables, J. G. 2004, MNRAS, 348, 1409

McLaughlin, D. E., Anderson, J., Meylan, G., et al. 2006, ApJS, 166, 249

Michelson, P. F., Bertsch, D. L., Brazier, K., et al. 1994, ApJ, 435, 218

O’Flaherty, K. S., Bennett, K., Diehl, R., et al. 1995, A\&A, 297, L29

Ransom, S. M. 2008, AIPC, 983, 415R

Rolland, L., Naurois, M., \& Raux, J. 2004, SF2A-Conf., 389

Stappers, B. W., Gaensler, B. M., Kaspi, V. M., van der Klis, M., \& Lewin, W. H. G. 2003, Science, 299, 1372

Tavani, M. 1991, ApJ, 379, L69

Venter, C., \& de Jager, O. C. 2008, ApJ, 680, L125

Venter, C., de Jager, O. C., \& Clapson, A.-C. 2009, ApJ, 696, L52

Wang, W., Jiang, Z. I., \& Cheng, K. S. 2005, MNRAS, 358, 263

Zavlin, V. E., Pavlov, G. G., Sanwal, D., et al. 2002, ApJ, 569, 894

${ }^{1}$ Max-Planck-Institut für Kernphysik, PO Box 103980, 69029 Heidelberg, Germany

e-mail: clapson@mpi-hd.mpg.de

2 Dublin Institute for Advanced Studies, 5 Merrion Square, Dublin 2, Ireland

3 Yerevan Physics Institute, 2 Alikhanian Brothers St., 375036 Yerevan, Armenia

${ }^{4}$ Universität Erlangen-Nürnberg, Physikalisches Institut, ErwinRommel-Str. 1, 91058 Erlangen, Germany

5 University of Durham, Department of Physics, South Road, Durham DH1 3LE, UK

${ }^{6}$ Centre d'Étude Spatiale des Rayonnements, CNRS/UPS, 9 Av. du Colonel Roche, BP 4346, 31029 Toulouse Cedex 4, France

7 Astroparticule et Cosmologie (APC), CNRS, Universite Paris 7 Denis Diderot, UMR 7164 (CNRS, Université Paris VII, CEA, Observatoire de Paris), 10 rue Alice Domon et Leonie Duquet, 75205 Paris Cedex 13, France

${ }^{8}$ Landessternwarte, Universität Heidelberg, Königstuhl, 69117 Heidelberg, Germany
9 Institut für Physik, Humboldt-Universität zu Berlin, Newtonstr. 15, 12489 Berlin, Germany

${ }^{10}$ LUTH, Observatoire de Paris, CNRS, Université Paris Diderot, 5 Place Jules Janssen, 92190 Meudon, France

11 IRFU/DSM/CEA, CE Saclay, 91191 Gif-sur-Yvette Cedex, France

12 Nicolaus Copernicus Astronomical Center, ul. Bartycka 18, 00-716 Warsaw, Poland

${ }^{13}$ Unit for Space Physics, North-West University, Potchefstroom 2520, South Africa

${ }^{14}$ Laboratoire d'Astrophysique de Grenoble, INSU/CNRS, Université Joseph Fourier, BP 53, 38041 Grenoble Cedex 9, France

15 LPNHE, Université Pierre et Marie Curie Paris 6, Université Denis Diderot Paris 7, CNRS/IN2P3, 4 Place Jussieu, 75252 Paris Cedex 5, France

${ }^{16}$ Laboratoire Leprince-Ringuet, École Polytechnique, CNRS/IN2P3, 91128 Palaiseau, France

${ }_{17}$ Laboratoire d'Annecy-le-Vieux de Physique des Particules, CNRS/IN2P3, 9 Chemin de Bellevue, BP 110, 74941 Annecy-leVieux Cedex, France

18 University of Namibia, Private Bag 13301, Windhoek, Namibia

19 Instytut Fizyki Ja̧drowej PAN, ul. Radzikowskiego 152, 31-342 Kraków, Poland

${ }^{20}$ Laboratoire de Physique Théorique et Astroparticules, CNRS/IN2P3, Université Montpellier II, CC 70, Place Eugène Bataillon, 34095 Montpellier Cedex 5, France

${ }^{21}$ Universität Hamburg, Institut für Experimentalphysik, Luruper Chaussee 149, 22761 Hamburg, Germany

${ }^{22}$ School of Physics \& Astronomy, University of Leeds, Leeds LS2 9JT, UK

${ }_{23}$ Institut für Astronomie und Astrophysik, Universität Tübingen, Sand 1, 72076 Tübingen, Germany

24 Toruń Centre for Astronomy, Nicolaus Copernicus University, ul. Gagarina 11, 87-100 Toruń, Poland

25 Institute of Particle and Nuclear Physics, Charles University, V Holesovickach 2, 18000 Prague 8, Czech Republic

26 European Associated Laboratory for Gamma-Ray Astronomy, jointly supported by CNRS and MPG

27 Obserwatorium Astronomiczne, Uniwersytet Jagielloński, ul. Orla 171, 30-244 Kraków, Poland

28 Stanford University, HEPL \& KIPAC, Stanford, CA 94305-4085, USA

${ }^{29}$ School of Chemistry \& Physics, University of Adelaide, Adelaide 5005, Australia

${ }^{30}$ Institut für Theoretische Physik, Lehrstuhl IV: Weltraum und Astrophysik, Ruhr-Universität Bochum, 44780 Bochum, Germany 